\title{
INVESTIGATION OF ANTIOXIDANT ACTIVITY AND CHEMICAL FINGERPRINT OF MARINE POLYCHAETE BASED ON ATR-FTIR METABOLOMICS
}

\author{
NUR IZZATI ZAMZAM ${ }^{1}$, NAZEEFA IZZATI KAMARUDIN ${ }^{1}$, IZWANDI IDRIS ${ }^{2}$, NOOR \\ ANIZA HARUN ${ }^{1}$, WAN IRYANI WAN ISMAIL ${ }^{1}$, RUDIYANTO ${ }^{3}$, FARIDAH ABAS ${ }^{4 *}$ AND \\ MAULIDIANI $^{1}$
}

\begin{abstract}
${ }^{1}$ Faculty of Science and Marine Environment, Universiti Malaysia Terengganu, 21030 Kuala Nerus, Terengganu, Malaysia. ${ }^{2}$ South China Sea Repository and Reference Centre, Institute of Oceanography and Environment (INOS), Universiti Malaysia Terengganu, 21030 Kuala Nerus, Terengganu, Malaysia. ${ }^{3}$ Faculty of Fisheries and Food Science, Universiti Malaysia Terengganu, 21030 Kuala Nerus, Terengganu, Malaysia. ${ }^{4}$ Department of Food Science, Faculty of Food Science and Technology, Universiti Putra Malaysia, Serdang 43400, Selangor, Malaysia.
\end{abstract}

*Corresponding author: faridah_abas@upm.edu.my

http://doi.org/10.46754/umtjur.2021.10.009

\begin{abstract}
Marine polychaete is an important component in marine benthic communities and can be widely found in Malaysia. To date, the information regarding its chemicals and biological activities of marine polychaete is still limited. This study aims to evaluate the chemical fingerprint of the marine polychaetes Marphysa moribidii and Diopatra claparedii using Attenuated Total Reflectance Fourier-Transform Infrared (ATR FTIR) metabolomics. The antioxidant activity including total phenolic content (TPC) and DPPH free radical scavenging activity was also evaluated. The results showed that $D$. claparedii has a higher amount of TPC $(0.47 \pm .0 .03 \mathrm{mg} \mathrm{GAE} / \mathrm{g})$ compared to $M$. moribidii $(0.30 \pm 0.01 \mathrm{mg} \mathrm{GAE} / \mathrm{g})$. The DPPH activity tested at the concentration $5000 \mu \mathrm{g} / \mathrm{ml}$ showed percentage of inhibition expressed by D. claparedii and M. moribidii extracts were 38.8011 .70 and 25.547.35 respectively. The principal component analysis (PCA) score plot showed distinct clusters between M. moribidii and D. claparedii. Further investigation via PCA loading plot showed that the FTIR signals at $3340,1090,1047$ and $880 \mathrm{~cm}^{-1}$ were contributed by D. claparedii. Meanwhile, the partial least square (PLS) analysis revealed several signals were correlated with the TPC including stretching vibration of $\mathrm{C}-\mathrm{H}$ at $2854 \mathrm{~cm}^{-1}, \mathrm{C}=\mathrm{C}$ bonds at $1640 \mathrm{~cm}^{-1}$ and $\mathrm{C}=\mathrm{C}$ bending at $1726 \mathrm{~cm}^{-1}$ showing the presence of aromatic ring deformations. This study provides the key important chemical fingerprint of different marine polychaetes (D. claparedii sp. and M. moribidii sp.) that might be useful for the discovery of bioactive compounds from natural resources.
\end{abstract}

Keywords: Marine polychaete, Marphysa moribidii, Diopatra claparedii, ATR-FTIR metabolomics, antioxidant.

\section{Introduction}

Polychaetes are widespread and most abundant in marine environment. It can be important for the eco-toxicology testing organisms according to their small size and short life cycles. The developmental stages and the life cycle characteristics have been found to be useful in providing more sensitive results for monitoring of pollutants (Pachiappan et al., 2015). Besides, marine polychaetes may have the potential as a source of bioactive compounds, but until now very limited information is recorded regarding its chemical constituents and biological activities. Earlier studies reported that marine polychaetes have been utilised for medical purposes including anti-oxidant and anti-woundhealing (Hussain et al., 2018; Nazri et al., 2019; Pei et al., 2020). Thus, a study needs to be done to determine the chemical profile of polychaetes as well as the biological activities. Several polychaete species can be found in Malaysia including Diopatra claparedii, D. neapolitana, Namalycastis rhodochorde, Vanadis minuta and Leiochrides australis (Fielman et al., 2001; Arshad \& Idris, 2013). Recently, a new marine polychaete Marphysa moribidii from the west coast of Peninsular Malaysia was reported (Idris et al., 2014). 
Metabolomics can be defined as the study of all metabolites in organisms that unravels the chemical and biological correlations (Verpoorte et al., 2007). Fourier-transformed infrared spectroscopy (FTIR) is one of the analytical tools that can be used in metabolomics research. Present study reported the chemical fingerprint of the two marine polychaetes (Marphysa moribidii and Diopatra claparediii) using FTIR metabolomics. In addition, the evaluation on their total phenolic content (TPC) and antioxidant activity was also carried out.

\section{Materials and Methods}

\section{Chemical Reagents}

Analytical organic solvents such as methanol and acetone were used for extraction and FTIR analysis. The chemicals 1, 1-diphenyl-2picryl-hydrazyl (DPPH), gallic acid and FolinCiocalteu were used for bioassay.

\section{Sample Collection}

D. claparedii sample was collected from the lower tidal flat zone of local mangrove during low tide and M. moribidii was collected at upper tidal flat zone of Pantai Kelanang, Morib mangrove in Selangor, Malaysia. The samples were ground and subjected to freeze dryer to remove excess water. The freeze-dried sample was kept at $-20^{\circ} \mathrm{C}$ prior to analysis.

\section{Sample Extraction}

About $100 \mathrm{mg}$ of freeze-dried sample in powder form was extracted with $1 \mathrm{~mL}$ methanol (ratio $1: 10 \mathrm{w} / \mathrm{v}$ ) and sonicated for $30 \mathrm{mins}$ at room temperature. The supernatant was filtered using $1 \mathrm{~mL}$ of syringe through a $0.45 \mu \mathrm{m}$ PTFE membrane filter (Nice $\left.{ }^{\circledR}\right)$. The extraction procedure was repeated for three times. Five individual extractions were performed to obtain five biological replicates for each polychaete species. The samples were concentrated under vacuum using rotary evaporator. The dried extracts were kept in the freezer $\left(4^{\circ} \mathrm{C}\right)$ prior to analysis.

\section{Total Phenolic Content}

Using the Folin-Ciocalteu method, the total phenolic content was performed (Lee et al., 2013). $20 \mu \mathrm{L}$ of the polychaete extracts $(3000$ $\mathrm{mg} / \mathrm{mL}$ concentration) were placed in 96-well microplate and $100 \mu \mathrm{L}$ of Folin-Ciocalteu reagent was added. The mixtures were incubated for five minutes. Then, $80 \mu \mathrm{L}$ of $7.5 \%$ sodium carbonate were added and incubated for two hours in the dark. The sample absorbance was measured at $750 \mathrm{~nm}$ using spectrophotometer. Both polychaete samples with five replicates were assayed and the results were presented as $\mathrm{mg}$ of gallic acid equivalents (GAE)/g of the sample based on a calibration curve prepared using gallic acid, assuming it is $100 \%$ pure (Lee et al., 2013).

\section{DPPH Free Radical Scavenging Activity}

The DPPH assay was used to ascertain the antioxidant activity of polychaete extracts. The test used a $50 \mu \mathrm{L}$ of sample solution at the concentration of $5000 \mu \mathrm{g} / \mathrm{mL}$ in $96-w e l l$ plate. Next, $100 \mu \mathrm{L}$ of DPPH $(60 \mu \mathrm{g} / \mathrm{mL})$ was added to each well. The reaction mixture was incubated at room temperature for 30 mins. A micro-plate reader was used to measure sample absorbance at $517 \mathrm{~nm}$. Five replicates were performed. The percentage inhibition was calculated as follow:

$\%$ inhibition $=[(\mathrm{AB}-\mathrm{AS}) / \mathrm{AB}] \times 100$

where,

$\mathrm{AB}$ and $\mathrm{AS}$ are the absorbance of reagent blank and tested samples, respectively.

The results are reported as mean \pm standard deviation of the percentage inhibition against DPPH at the concentration of $5000 \mu \mathrm{g} / \mathrm{ml}$. Gallic acid was used as positive control (Abd Ghafar et al., 2018).

\section{ATR-FTIR Analysis}

Infrared spectra were obtained from Nicolet ${ }^{\mathrm{TM}}$ iS ${ }^{\mathrm{TM}} 10$ FTIR Spectrometer (Thermo Fisher Scientific, Madison, WI, USA). About 0.1 $\mathrm{mL}$ of the sample $(1 \mathrm{mg} / \mathrm{ml})$ was loaded onto 
ATR crystal centre. Samples were dried for approximately $40 \mathrm{~s}$ at room temperature. Then, each spectrum was recorded between the wavelengths of $4000-600 \mathrm{~cm}^{-1}$ with 40 interferograms at a resolution of $4 \mathrm{~cm}^{-1}$. Before each sample scan, a spectrum of the ATR crystal was recorded using the same instrumental conditions as background. The ATR plate was cleaned using acetone and a dust-free tissue after each sample scan. For FTIR analysis of the samples, five biological replicates with three technical replicates were performed. All FTIR data were converted to ASCII files and subjected to multivariate data analysis.

\section{Multivariate Data Analysis (MVDA)}

The multivariate data analysis (MVDA) including component analysis (PCA) and partial least square (PLS) were carried out using the SIMCA-P software (v. 14.1, Umetrics, Umeå, Sweden) with Pareto scaling applied to all the data. The PCA is a technique to reduce the dimensionality of such datasets, to increase interpretability but at the same time to minimize information loss. It is usually used for the metabolite fingerprint and classification purposes. In this study, the PLS model was used to evaluate the relationship between the chemical fingerprints from FTIR data with its total phenolic content (TPC) and DPPH activity. Partial least square model is often used in metabolomics, where predictive variables consist of many different measurements in an experiment (Maulidiani et al., 2013).

\section{Results and Discussion}

\section{Total Phenolic Content (TPC) and DPPH Activity of D. claparedii and M. moribidii}

Table 1 showed the TPC and DPPH activity of D. claparedii and M. moribidii. The methanolic extract of D. claparedii showed higher TPC (0.47 mg GAE/g) compared to M. moribidii (0.30 mg GAE $/ \mathrm{g})$. The same pattern was also observed for the DPPH activity. The DPPH result of $D$. claparedii showed higher antioxidant activity compared to M. moribidii. The positive correlation was observed between DPPH activity and TPC of the marine polychaetes, and it can be concluded that TPC might have contributed to the DPPH activity.

Table 1: Total phenolic content (TPC) and DPPH activity of D. claparedii and M. moribidii

\begin{tabular}{ccc}
\hline Species & TPC $(\mathbf{m g ~ G A E} / \mathbf{g}$ sample) & DPPH Activity (at $\mathbf{5 0 0 0} \boldsymbol{\mu g} / \mathbf{m L})$ \\
\hline D. claparedii & $0.47 \pm 0.03$ & 38.8011 .70 \\
M. moribidii & $0.30 \pm 0.01$ & 25.547 .35 \\
\hline
\end{tabular}

\section{FTIR spectra of D. claparedii and M. moribidii}

In general, the two polychaetes showed the same pattern based on their FTIR spectra (Figure 1). In spite of that, the different intensity in the wavenumbers can be observed in their FTIR spectra. The FTIR spectra of $D$. claparedii and M. moribidii showed characteristic band with strong intensity at $1023 \mathrm{~cm}^{-1}$ resulting from C-O stretching (Figure 1). In addition, band in the area of $1391 \mathrm{~cm}^{-1}$ corresponding to $\mathrm{CH}_{3}$ bending while band of $1700 \mathrm{~cm}^{-1}$ with weak intensity was also seen due to the $\mathrm{C}=\mathrm{O}$ (amide). The signals at $2872-2965 \mathrm{~cm}^{-1}$ correspond to $\mathrm{CH} \mathrm{sp} 3$ stretching for both symmetric signals at 2850 and asymmetric at $2920 \mathrm{~cm}^{-1}$, respectively. The aforementioned are the characteristic signals of polysaccharides, lipids, and carbohydrates. In addition, a broad band in a range of $3055-3626 \mathrm{~cm}^{-1}$ with medium intensity is attributed to the functional groups of $\mathrm{O}-\mathrm{H}$ and $\mathrm{N}-\mathrm{H}$ stretch (water, alcohols, phenols, and amine). Detail identification of possible compounds in marine polychaete is presented in Table 2. In order to gain a clearer overview on the chemical fingerprint of the polychaete samples, the multivariate data analysis of PCA was conducted. 


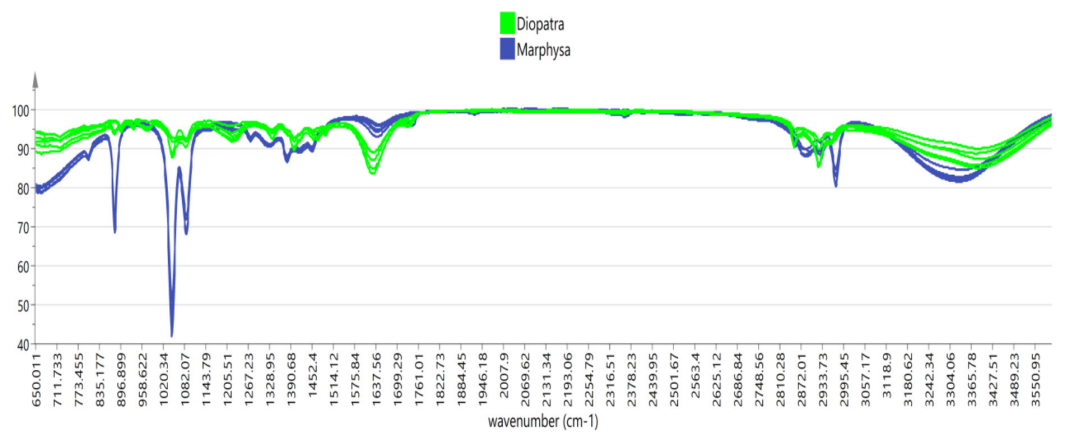

Figure 1: ATR-FTIR spectra overlay of $D$. claparedii and M. moribidii

Table 2: Tentative identification of compounds in marine polychaetes based on FTIR data

\begin{tabular}{|c|c|c|c|}
\hline $\begin{array}{l}\text { Wavenumber } \\
\text { Range }\left(\mathbf{c m}^{-1}\right)\end{array}$ & Function Groups & Tentative Assignment & Reference(s) \\
\hline $3055-3626$ & $\mathrm{O}-\mathrm{H}$ and $\mathrm{N}-\mathrm{H}$ stretching & Alcohols, phenols, amine & $\begin{array}{l}\text { Pei et al. (2020), Anguebes } \\
\text { et al. (2016), Oliveira et al. } \\
\text { (2016) }\end{array}$ \\
\hline $2872-2965$ & $\mathrm{C}-\mathrm{H}$ stretching & $\begin{array}{l}\text { Carbohydrates, lipids, } \\
\text { phenols }\end{array}$ & Oliveira et al. (2016) \\
\hline $1570-1762$ & $\begin{array}{l}\mathrm{N}-\mathrm{H} \text { bending vibrations, } \\
\mathrm{C}=\mathrm{O} \text { bending vibrations }\end{array}$ & $\begin{array}{c}\text { Amino acids, fatty acids, } \\
\text { ester }\end{array}$ & $\begin{array}{c}\text { Pei et al. (2020), Oliveira } \\
\text { et al. (2016) }\end{array}$ \\
\hline $1315-1500$ & $\mathrm{CH}$ and $\mathrm{OH}$ bending & $\begin{array}{l}\text { Alkene, carboxylic acids, } \\
\text { alcohols, phenols }\end{array}$ & $\begin{array}{c}\text { Pei et al. (2020), Oliveira } \\
\text { et al. (2016) }\end{array}$ \\
\hline $1250-1020$ & $\mathrm{C}-\mathrm{N}$ and $\mathrm{C}-\mathrm{O}-\mathrm{C}$ stretching & Amine, carbohydrates & Anguebes et al. (2016) \\
\hline $1045-1089$ & $\mathrm{C}-\mathrm{O}$ and $\mathrm{S}=\mathrm{O}$ stretching & $\begin{array}{l}\text { Aliphatic ether, primary } \\
\text { and secondary alcohols, } \\
\text { sulfoxide }\end{array}$ & Anguebes et al. (2016) \\
\hline $898-980$ & $\mathrm{C}=\mathrm{C}$ bending & Alkene & Oliveira et al. (2016) \\
\hline 880 & $\mathrm{C}-\mathrm{H}$ bending & $\begin{array}{c}\text { 1,2,4-trisubstituted, } \\
\text { 1,3-disubstituted }\end{array}$ & Oliveira et al. (2016) \\
\hline $650-680$ & $\mathrm{C}-\mathrm{Br}$ stretching & Halogen compounds & Pei et al. (2020) \\
\hline
\end{tabular}

\section{Principal Component Analysis (PCA)}

While there were strong visual variations between the spectra, multivariate analysis was used to further evaluate the samples for a nonbiased interpretation of the findings. Principal component analysis (PCA) is one of the methods most widely used among statistical methods. In this study, the PCA model was constructed using all the analytical samples. The first two PC's explained variance of $87.33 \%$. The PCA is considered to be a good model with values of
R2X (cum) and Q2 (cum) of 0.873 and 0.797, respectively. The score plot serves to identify any groupings in the data set while the loading plots can be used to identify the wavenumbers that are responsible for the separation of the samples. Figure 2 showed the PCA score plot of the two polychaete samples generated based on its FTIR. It showed a clear discrimination between $D$. claparedii and M. moribidii extracts. The loading plot (Figure 3) showed that the wavenumbers at 3340, 2940, 2854, 1090, 1047 
and $880 \mathrm{~cm}^{-1}$ were the characteristics of $D$. and $1640 \mathrm{~cm}^{-1}$ appeared to be more prominent in claparedii while the wavenumbers at 2900,1726 M. moribidii.

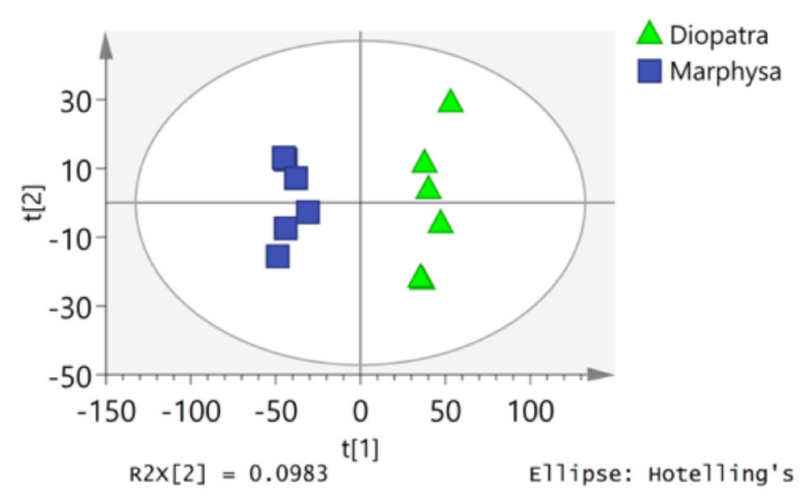

Figure 2: Principal component analysis (PCA) score plot of D. claparedii versus $M$. moribidii

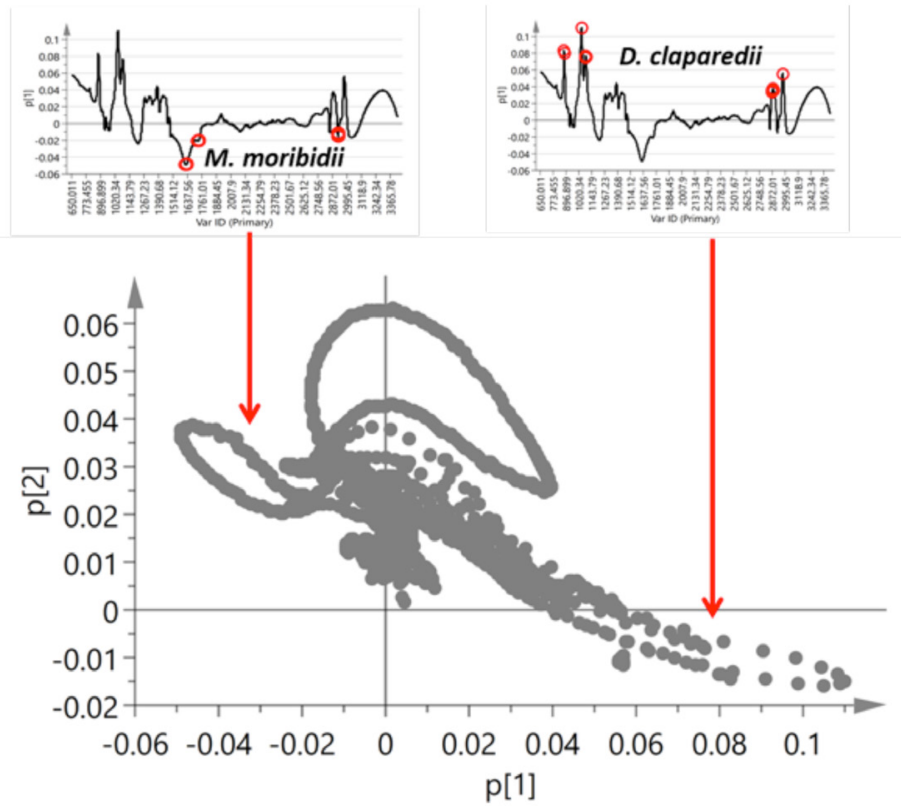

Figure 3: Principal component analysis (PCA) loading plot of D. claparedii versus M. moribidii

\section{Partial Least Square (PLS) Analysis}

PLS model was conducted to evaluate the relationship between the FTIR signals with TPC and DPPH activity. The $\mathrm{X}$ variables represented the wavenumbers, while the $\mathrm{Y}$ variables represented TPC and DPPH activity. The results are presented in PLS biplot, a single graphic representation was produced, combining the score and the loading plots.

A distinct difference between the two types of polychaete species, D. claparedii and M. moribidii, was observed based on the PLS biplot (Figure 4). The PLS analysis revealed several wavenumbers correlated with the TPC 
and $\mathrm{DPPH}$ activity including $\mathrm{C}=\mathrm{C}$ and $\mathrm{C}=\mathrm{O}$ vibration at $1631 \mathrm{~cm}^{-1}$, bending $(\delta)$ vibration of $\mathrm{C}-\mathrm{H}$ and the stretching vibration of aromatics $(-\mathrm{C}=\mathrm{C}-)$ at $1400 \mathrm{~cm}^{-1}$, and signal at $881 \mathrm{~cm}-1$ due to aromatic ring vibration (Oliveira et al., 2016). The signal at $2926 \mathrm{~cm}^{-1}$ indicated the presence of C-H stretching vibration of methyl and methoxy groups and stretching vibration of $-\mathrm{CH} 3$ or $-\mathrm{CH} 2$ groups in carboxylic acid. In addition, the $\mathrm{OH}$ wagging ( $\mathrm{OH}$ of phenolics) can be identified based on the FTIR signal at $3379 \mathrm{~cm}^{-1}$ (Oliveira et al., 2016). Furthermore, the TPC and DPPH activity positioned at the same quadrant in the right side of PLS biplot indicated that the TPC has positive correlation with DPPH activity. Thus, it can be concluded that the antioxidant activity of polychaetes was closely related to the amount of phenolics. This finding is in agreement with a previous study which reported that TPC showed positive correlation to DPPH activity (Abd Ghafar et al., 2018).

The PLS model was shown to be a good model indicated by its R2Y (cum) value of 0.602. Although the Q2 (cum) value of 0.325 is slightly low, the validity of PLS model was confirmed by permutation test and supported by the results from observed and predicted plots. The permutation test result for TPC showed R2 and Q2 values of 0.106 and -0.239 , respectively. Meanwhile the permutation test results for DPPH activity showed R2 and Q2 values of 0.33 and -0.243 , respectively. Both TPC and DPPH activity of observed and predicted plots showed $\mathrm{R} 2$ values $>0.5$. Thus, the PLS model used in this study met the criteria of good model and can be used for prediction purposes.

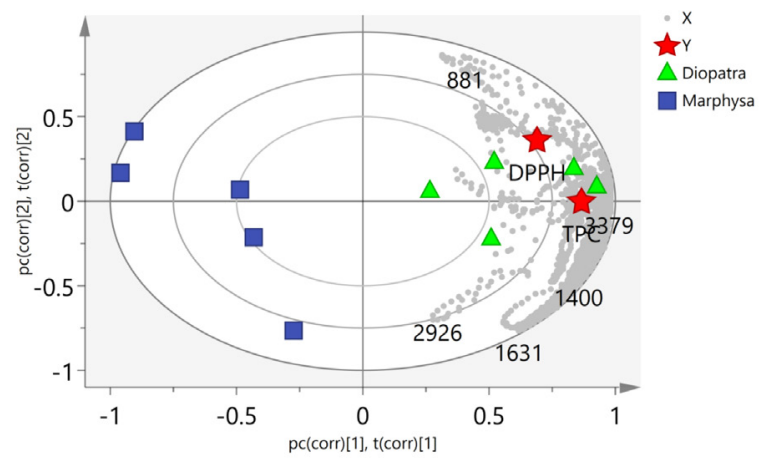

Figure 4: PLS Bi-Plot of D. claparedii versus $M$. moribidii correlates with its TPC and DPPH activity; The wavenumbers at 3379, 2976, 2926, 1631, 1400 and $881 \mathrm{~cm}^{-1}$ correspond to TPC and DPPH activity

\section{Conclusion}

The ATR-FTIR metabolomics was successfully applied to determine the chemical profile of two marine polychaete species (D. claparedii and M. moribidii). Both polychaetes showed weak antioxidant activity. The PLS model showed some FTIR signals at 3379, 2976, 2926, 1631, 1400 and $881 \mathrm{~cm}^{-1}$ were positively correlated to its TPC and DPPH activity. The outcome of this study provides preliminary information on the chemical profiles and biological activity of marine polychaetes in Malaysia.

\section{Acknowledgements}

We would like to thank the Faculty of Science and Marine Environment, Universiti Malaysia Terengganu for the support under the Final Year Project (FYP) research funding. Our appreciation is also extended to the Faculty of Fisheries and Food Science, Universiti Malaysia Terengganu for providing the ATR-FTIR instrument. 


\section{References}

Abd Ghafar, S., Mediani, A., Maulidiani, Ramli, N., \& Abas, F. (2018). Antioxidant, $\alpha$-glucosidase, and nitric oxide inhibitory activities of Phyllanthus acidus and LCMS/MS profile of the active extract. Food Bioscience, 25, 134-140.

Anguebes, F., Pat, L., Ali, B., Guerrero, A., Córdova, A. V., Abatal, M., \& José P. Garduza, J. P. (2016). Application of multivariable analysis and FTIR-ATR spectroscopy to the prediction of properties in Campeche honey. Journal of Analytical Methods in Chemistry, 5427526, 1-14.

Arshad, A., \& Idris, I. (2013). Checklist of polychaetous annelids in Malaysia with redescription of two commercially exploited species. Asian Journal of Animal and Veterinary Advances, 8(3), 409-436.

Fielman, K. T., Lincoln, D. E., \& Woodin, S. A. (2001). Polychaete indicator species as a source of natural halogenated organic compounds in marine sediments. Environmental Toxicology and Chemistry: An International Journal, 20(4), 738-747.

Hussain, N. S., Harun, N. A., Mohd Radzi, M. N. F., Idris, I., \& Ismail, W. I. W. (2018). Biosynthesis of silver nanoparticles from marine polychaete Diopatra claparedii Grube, 1878. Jurnal Teknologi (Sciences \& Engineering), 80(6), 181-187.

Idris, I., Hutchings, P., \& Arshad, A., 2014. Description of a new species of Marphysa quatrefages, 1865 (Polychaeta: Eunicidae) from the west coast of Peninsular Malaysia and comparisons with species from Marphysa Group A from the Indo-West Pacific and Indian Ocean. Memoirs of Museum Victoria, 71, 109-121.
Lee, S.Y., Mediani, A., Ismail, I. S., Maulidiani., \& Abas, F. (2019). Antioxidants and $\alpha$-glucosidase inhibitors from Neptunia oleracea fractions using ${ }^{1} \mathrm{H}$ NMR-based metabolomics approach and UHPLC-MS/ MS analysis. BMC Complementary and Alternative Medicine, 19(7), 2019.

Maulidiani, Abas, F., Khatib, A., Shitan, M., Shaari, K., \& Lajis, N. H. (2013). Comparison of partial least squares and artificial neural network for the prediction of antioxidant activity in extract of Pegaga (Centella) varieties from ${ }^{1} \mathrm{H}$ nuclear magnetic resonance spectroscopy. Food Research International, 54, 852-860.

Nazri, M. U. I. A., Idris, I., Ross, O., \& Ismail, W.I.W. (2019). Neurological disorder brain model: A lesson from marine worms (Annelida: Polychaeta). Malaysian Journal of Medical Sciences, 26(6), 5-18.

Oliveira, R. N., Mancini, M. C., de Oliveira, F. C. S., Passos, T. M., Brid Quilty, B., Thiré, R. M. D. M., \& McGuinness, G. B. (2016). FTIR analysis and quantification of phenols and flavonoids of five commercially available plants extracts used in wound healing. Revista Matéria, 21(3), 767-779.

Pachiappan, P., Perumal, S., \& Thirunavukkarasu, A. R. (Eds.). (2015). Advances in marine and brackishwater aquaculture. Springer, India.

Pei, A. U. E., Piong, C. H., Masimen, M. A. A., Ismail, W. I. W., Idris, I., \& Harun, N. A. (2020). Biosynthesis of gold nanoparticles (AuNPs) by marine baitworm Marphysa moribidii Idris, Hutchings and Arshad 2014 (Annelida: Polychaeta) and its antibacterial activity. Advances in Natural Sciences: Nanoscience and Nanotechnology, 11(1), 015001.

Verpoorte, R., Choi, Y., \& Kim, H. (2007). NMR-based metabolomics at work in phytochemistry. Phytochemistry Reviews, 6(1), 3-14. 
